\title{
Heating up a lantern with a tealight candle
}

\author{
P. Wattanakasiwich, P. Kongkhumbod and N. Pussadee \\ Department of Physics and Materials Science, Faculty of Science, \\ Chiang Mai University, Chiang Mai, 50200 Thailand.
}

Received 10 February 2021; accepted 26 March 2021

\begin{abstract}
This paper provides physics principles and a method to heating up a lantern with a tealight candle, so it reaches $2.5 \mathrm{~m}$ within the shortest time. The experiments aimed to determine optimal parameters in filling paper lanterns with hot air and the ideal shape of lanterns that would travel most quickly in a vertical direction. Hot air from burning a 28 -wick candle was directed through a heat transfer system to fill the lanterns. The small ellipsoid lantern required the shortest time. This problem is suitable as a platform for STEM education approach on topics of convection, buoyancy and drag force.
\end{abstract}

Keywords: Paper lanterns; drag force; buoyancy; lifting force; convection rate; tealight candle; an ill-structured problem; STEM education.

DOI: https://doi.org/10.31349/RevMexFisE.19.010206

\section{Introduction}

STEM education is an approach to teaching and learning that integrates the content and skills in four specific disciplines science, technology, engineering and mathematics - in a cohesive learning paradigm based on real-world applications. STEM education can be based on problem-based or projectbased learning (PBL). This is one form of inquiry to empower learners to conduct experiment or study, integrate theory and practice, and apply knowledge and skills to develop a viable solution to an ill-structured problem [1].

In this paper, we proposed a solution to a problem called "Lantern" from International Young Physicist Tournament in 2012 as the problem for PBL. The problem [2] states that "Paper lanterns float using a candle. Design and make a lantern powered by a single tea-light that takes the shortest time (from lighting the candle) to float up a vertical height of $2.5 \mathrm{~m}$. Investigate the influence of the relevant parameters." In this study, we provided related physics principles, designed a convective heat transfer system, and conducted experiments to determine optimal parameters in heating up and floating the lanterns.

\section{Related physics principles}

Floating lanterns can be explained using the physics of hot air balloons $[3,4]$. Air that has a higher temperature than that of its surroundings generates buoyancy causing both hot air balloons and floating lanterns to rise. However, the difference between these two objects is that hot air balloons have fuel source attached to them even as they rise whereas the floating lanterns presented in this question do not. The relevant forces prior to release include the lift force $\left(F_{l i f t}\right)$ and the weight of the lantern $(M g)$, once the lantern has begun rising an additional drag force $\left(F_{D}\right)$ must be considered.

\subsection{Conditions for lantern to rise}

The lift force is the resultant force vector of the buoyancy force and the mass of the hot air in the lantern. When an object sinks or floats in a fluid (liquid or gas), the fluid exerts a force on the object called the buoyancy force. The fluid in this system is the air within the lantern which has a higher temperature than outside it and therefore has an effect on the system. Following Archimedes' Principle, the magnitude of the buoyancy force, $F_{B}$ is equal to the cold air weight that has been displaced by the lantern. This weight is a product of cold air density, $\rho$, the volume of the lantern, $V$, and the acceleration due to gravity, $g$ :

$$
F_{B}=\rho V g .
$$

Given the buoyancy force which has an upward direction and the weight of the hot air inside the lantern, $m g$, which has a downward direction, the resultant force vector is given by:

$$
F_{\text {lift }}=F_{B}-g .
$$

By substituting the expression for the buoyancy force Eq. (1) into Eq. (2) and the mass and density of the hot air, $\rho_{\text {hot }}$ the lifting force equation becomes:

$$
F_{\text {lift }}=\left(\rho_{\text {hot }}-\rho\right) V g .
$$

The lantern will begin to rise once $F_{\text {lift }}>M g$. By substituting in Eq. (3), the condition becomes

$$
\rho_{\text {hot }}-\rho>\frac{M}{V} .
$$

The density of the surrouding air can be written in terms of pressure, $P$ and temperature, $T$, with the specific gas constant, $R_{s}=287.058 \mathrm{~J} / \mathrm{kg} \mathrm{K}$, for dry air.

$$
\rho=\frac{P}{R_{s} T},
$$


can be substituted into Eq. (4), and so the temperature of the air in the lantern required to make it float $\left(T_{\text {hot }}\right)$ can be found from this equation.

$$
T_{\text {hot }}>\frac{P T}{P-\left(\frac{M}{V}\right) R_{s} T} .
$$

Equation (6) shows that the lantern can only rise if the temperature in the lantern is larger than $T_{\text {hot }}$ which is dependent on the ambient temperature, the ambient air pressure, and the ratio of the lanterns mass to its volume $(M / V)$.

\subsection{Conditions for maximum velocity}

To maximize lantern velocity there must be as little drag force as possible because drag force always has a direction opposite to that of the movement of the object. In the case of large objects falling through the air such as a balloon in free fall or a parachutist, the drag force changes with the velocity, $v$. Drag force depends on the air density, $\rho$, the cross-sectional area of the object, $A$ and the drag coefficient of the moving object in air due to its shape, $C_{D}$.

$$
F_{D}=\frac{1}{2} C_{D} \rho A v^{2}
$$

When the floating lantern is released and begins to travel upwards, the drag force acts downwards, the magnitude of the drag force is dependent on the density of the surrounding air in that moment and the shape of the lantern. Lanterns with different shapes will have different cross-sectional areas and therefore different drag coefficients. Table I shows the drag coefficients of different shapes. Ellipsoids have the lowest $C_{D}$ and should therefore take the least amount of time from release to travel $2.5 \mathrm{~m}$ if the initial velocity is the same.

\subsection{Conditions for minimizing time elapsed}

This problem has a condition that the lantern has to travel 2.5 $\mathrm{m}$ in the least time possible, beginning the moment the fuel source is lit. The total amount of time $\left(t_{\text {total }}\right)$ consists of the

TABLE I. Drag coefficients of different shapes.

\begin{tabular}{cc}
\hline & $\begin{array}{c}\text { Drag Coefficient } \\
\left(C_{D}\right)[5]\end{array}$ \\
Cylindrical & 0.82 \\
Cuboid & 1.05 \\
Streamlined-body & $3.43 \times 10^{-2}$ \\
Ellipsoid & $2.48 \times 10^{-2}$ \\
\hline
\end{tabular}

time taken to heat up the air in the lantern $\left(t_{\text {heat }}\right)$ and the time the lantern takes to travel $2.5 \mathrm{~m}$ vertically $\left(t_{\text {float }}\right)$. As such, to solve this problem, factors that would reduce the time take in each section must be considered. The factors that would affect each time phase are as follows:

- $t_{\text {heat }}$ is affected by the rate at which the fuel source releases heat into the lantern which in turn affects the inside temperature required according to Eq. (6). The relevant parameters to reduce the time taken in this section include convective heat transfer rate into the lantern, the shape of the lantern, and the value of $T_{\text {hot }}$.

$t_{\text {float }}$ is dependent on the shape of the lantern; choosing the one with the smallest drag coefficient should minimize the time taken.

\section{Experimental setup}

The experiment was split into 3 sections to construct of a more effective convective heat transfer system, determine the shape of the lantern that took the least time to heat up, and determine the most aerodynamic shape.

\subsection{Heat transfer system and heat source}

Heat from burning candle was being transferred in all directions causing the air around the candle to be heated up and expand in all directions. As such, to ensure that heat transfer occurred in a single direction and the particles with high temperatures travelled up into the lantern a delivery system was devised as shown in Fig. 1a) consisting of:

- A reflector taken from a lamp shade made of aluminum which reflected $96 \%$ of light. This material can also reflect electromagnetic waves at other frequencies in addition to visible light making it possible to be used to direct heat in certain directions as shown in Fig. 1b).
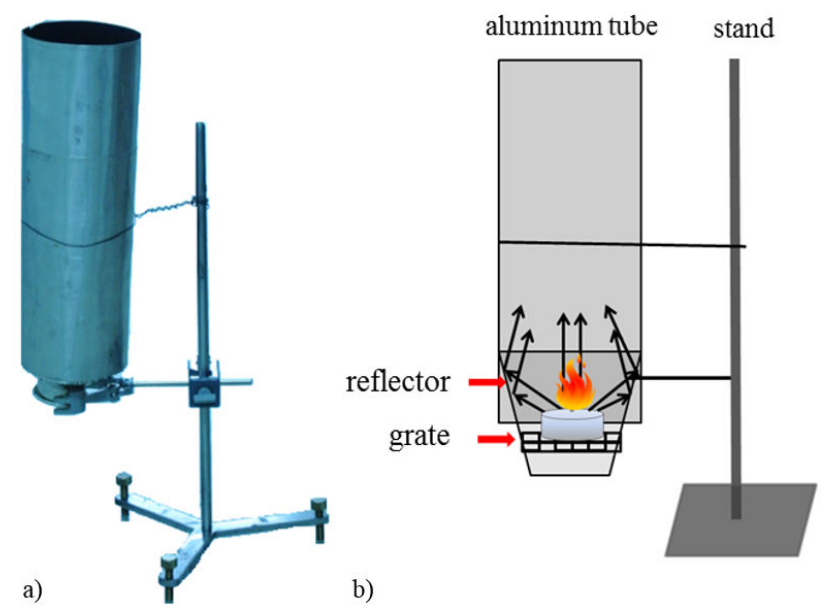

FIGURE 1. a) Images showing the heat transfer system and b) diagram showing the equipment used in the heat transfer system and the reflection of the electromagnetic waves (black arrows) inside it. 


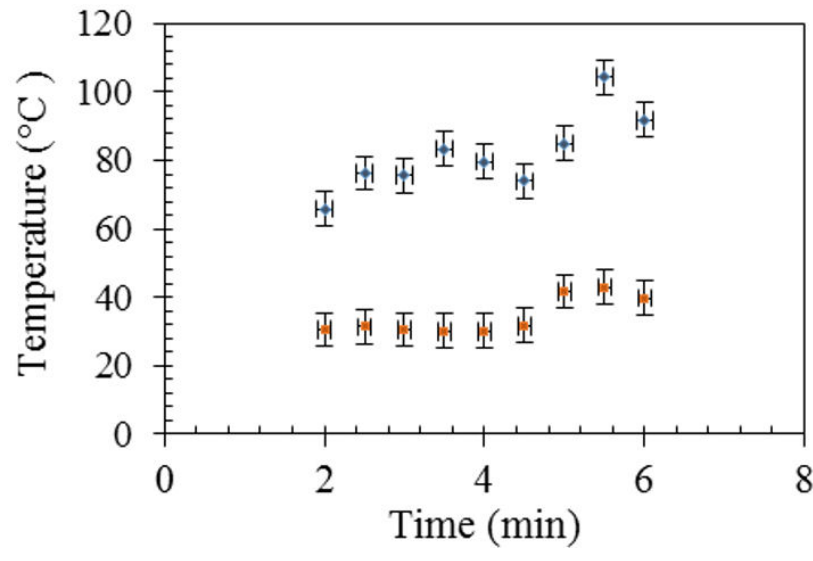

FIGURE 2. Temperature measuring with a thermocouple at $30 \mathrm{~cm}$ above the candle with the heat transfer system $(\bullet)$ and without the system ( $\square$ ).

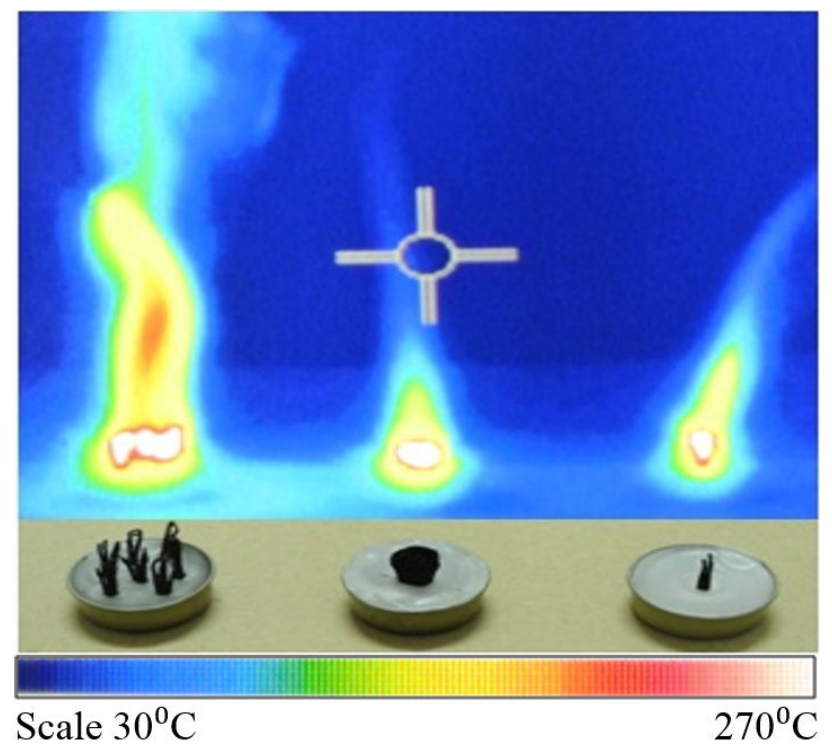

FIGURE 3. IR image showing the temperature of candles with (from left to right) 28 wicks spread out evenly, 28 wicks grouped to together, and a single wick.

- An aluminum tube to direct the flow of high temperature particles that are rising into the lantern to increase the convective heat transfer rate from candle to lantern.

- A grate upon which to place the candle and allow air flow into the tube and therefore providing consistent air flow to stimulate convection as well as provide oxygen from below the grate for the combustion process.

- A stand to secure all the equipment in vertical alignment.

The developed heat transfer system theoretically increased the convective heat transfer rate into the lantern, shortening $t_{\text {heat }}$. To prove the efficacy of the system, an experiment was devised where a traditional lantern would be heated up with a single wick candle with and then without the heat transfer system. Temperature readings were taken at $30 \mathrm{~cm}$ above the candle with and without the heat transfer system using a thermocouple beginning at 2 minutes after the candle was first lit until 6 minutes at 30 second intervals.

From Fig. 2, it can be seen that the temperature readings at $30 \mathrm{~cm}$ above the candle are significantly different when compared them with and without the heat transfer system. The difference in temperature between the two experiments ranged between $30-60^{\circ} \mathrm{C}$ with the heat transfer system having significantly higher temperatures. Additionally, using the heat transfer system reduced the risk of the lantern coming into contact with the flame making the heating process significantly safer with than without it.

The problem required the use of a tea light candle as a heat source when heating up the lantern, tea lights being candles in a small, cylindrical container with a diameter and height of approximately 1.5 and 0.6 inches [6]. However, components within tea lights that are not restricted by standardization include the composite, number of wicks, shape of the container, etc. The researcher therefore made paraffin tea lights themselves to be able to control the number of wicks used and their placements and therefore find the candle that produced the most heat.

Candle wicks are made from twined cotton or nylon fibers to create a string which is then treated with a chemical to make them more resistant to burning and able to stand up straight. Wicks have the important task of transferring the melted paraffin to where the flame is using capillary action which occurs when the adhesive forces between the molecules of the liquid paraffin and wick fibers surpass the cohesive forces between the paraffin molecules. As such, the number of wicks affects the amount of heat produced; increasing the number of wicks increases the combustion efficiency of the candle and therefore increases the amount of heat produced [7].

Figure 3 displays infrared (IR) pictures taken using a Flir i7 Thermal Imaging Camera to collect data about temperatures at the candle and area surrounding the flame, it was found that candles with wicks spread out across a large area produce more heat. In Fig. 3, the candle on the left with evenly spread out 28 wicks had a higher temperature and heated up a larger volume of air surrounding the candle, increasing the convective heat transfer rate and was therefore chosen for use with the heat transfer system in further experimentation.

\subsection{Most quickly heated lantern shape}

This experiment was intended to find the shape of lantern that took least amount of time for the temperature to surpass $T_{\text {hot }}$ which is required for the lantern to rise up; however $T_{\text {hot }}$ is dependent on the ambient temperature and pressure as well as the mass to volume ratio of the lantern $(M / V)$. Assuming that the ambient temperature and pressure are constant, $M / V$ becomes the only variable that has an effect on $T_{\text {hot }}$. As such, the shape with the lowest $M / V$ would result in the lowest 
TABLE II. Display mass, volume and $M / V$ value for different shapes and calculated Thot based on Eq. (6).

\begin{tabular}{ccccc}
\hline Lantern Shape & $M \pm 0.05(\mathrm{~g})$ & $V \pm 0.001\left(\mathrm{~m}^{3}\right)$ & $M / V \pm 0.001\left(\mathrm{~kg} / \mathrm{m}^{3}\right)$ & $T_{\text {hot }}\left({ }^{\circ} \mathrm{C}\right)$ \\
\hline Traditional & 28.70 & 0.159 & 0.181 & $85.8 \pm 7.7$ \\
Cuboid & 32.28 & 0.159 & 0.203 & $94.4 \pm 8.0$ \\
Spherical & 34.12 & 0.159 & 0.215 & $99.3 \pm 8.3$ \\
Small Ellipsoid & 27.70 & 0.159 & 0.174 & $83.1 \pm 7.5$ \\
Large Ellipsoid & 46.25 & 0.259 & 0.179 & $85.0 \pm 7.6$ \\
\hline
\end{tabular}

$T_{\text {hot }}$ meaning that the shape would require the lowest temperature to rise and would therefore have the lowest $t_{\text {heat }}$.

In the experiment, lantern paper was used to make different shaped lanterns, specifically: traditional, cuboid, spherical, small ellipsoid, and large ellipsoid. The lanterns created were weighed to 2 significant figures and had their volume measured by completely filling up the lantern with small, Styrofoam filler beads and finding the amount of volume the beads took up. This process allowed the ratio $M / V$ to be calculated using Eq. (6) for the different shaped lanterns with results as shown in Table I. The results indicated that the small ellipsoid lantern would have the lowest $T_{\text {hot }}$.

The atmospheric pressure was measured using a Vernier Pressure Sensor to find $P=96.5 \mathrm{kPa}$, a Vernier Temperature Sensor was used to measure the atmospheric temperature to find $T=300.8 \mathrm{~K}$, Eq. (6) was used to calculate $T_{\text {hot }}$ for the different shaped lanterns shown in Table II.

From Table II, the small ellipsoid lantern had the lowest calculated $T_{\text {hot }}$ at $83.1^{\circ} \mathrm{C}$ while the spherical one had the highest at $99.3^{\circ} \mathrm{C}$. To compare the theoretical and experimental values of $T_{\text {hot }}$, traditional, cuboid, spherical, small ellipsoid, and large ellipsoid lanterns were created and then heated up using candles with multiple, spread out wicks and the convective heat transfer system. IR images were taken using the Flir i7 Thermal Imaging Camera to measure the temperatures of each lantern at 60 second intervals from $60-240$ seconds with an additional reading at 270 seconds beginning when the candles were first lit. A digital IR thermometer was used to measure temperature at the top of each lantern. When this temperature reached $T_{\max }$, the lantern was released to float.

\subsection{Most aerodynamic lantern shape}

This experiment aimed to determine the lantern shape that was the most aerodynamic and therefore would take the least amount of time to travel $2.5 \mathrm{~m}$ once the lantern had been heated and begun floating. The total amount of time taken consists of the time taken to heat up the lantern until it lifts off, $t_{\text {heat }}$, and the amount of time from lift off to until the lantern travels $2.5 \mathrm{~m}, t_{\text {float }}$. Based on the basic principles of aerodynamics, an ellipsoid object would have the lowest coefficient of drag as shown in Table I. As such, an ellipsoid lantern should take the least amount of time to travel $2.5 \mathrm{~m}$, assuming the take-off velocity was the same for all shapes.
The equipment used in this experiment included the convective heat transfer system, a multi-wick candle, and apparatus to ensure a straight flight trajectory of the lantern.

\section{Results and discussion}

We had designed an effective convective heat transfer rate that would allow the temperature of the air in the lantern to reach $T_{\text {hot }}$ within a short time frame. Then, this system combined with a 28 -wick candle was a fuel source for increasing air temperature inside the lantern. IR images were taken at $60,120,180,240$, and 270 seconds after the candle was lit for each type of lantern and presented in Table III to show the progression of temperature distribution when the heat transfer rate was approximately the same for each lantern. The last column in the table displays the highest temperatures, $T_{\max }$ reached by different shapes; the traditional lantern reached the highest temperature while the spherical lantern reached the lowest. However, the small ellipsoid lantern had the most consistent heat distribution throughout the lantern. When comparing these results with the $T_{\text {hot }}$ values calculated using Eq. (6) in Table II, we find that the small ellipsoid lantern also had one of the lowest $T_{\text {hot }}$ values.

Table II shows the predicted minimum temperature or $T_{\text {hot }}$ each lantern must reach to begin floating with the small ellipsoid lantern required the lowest $\left(83.1^{\circ} \mathrm{C}\right)$ and the spherical lantern required the highest $\left(99.3^{\circ} \mathrm{C}\right)$. When these values are taken into consideration alongside the IR images from Table III, at approximately 210 - 240 seconds, the traditional and small ellipsoid lanterns reached high enough temperature to begin floating $\left(T_{\max }>T_{\text {hot }}\right)$. The small ellipsoid-shaped lantern had the most consistent high temperature distribution throughout the lantern indicating that its average temperature was higher than the rest. Meanwhile the spherical, cuboid and large ellipsoid lantern required more than $270 \mathrm{~s}$ to be filled with enough hot air to allow it to float. It could be concluded that the small ellipsoid lantern was the appropriate choice as it required the least time to be filled with air hotter than the $T_{\text {hot }}$ value relevant to the shape shown by Table IV. However, the standard error of mass to volume $M / V$ which is then used to calculate the value of $T_{\text {hot }}$ resulting in a standard error of 7.5 to $8.3^{\circ} \mathrm{C}$ which could in fact result in a negligible difference in $T_{\text {hot }}$ between the traditional, small ellipsoid, and large ellipsoid lanterns. 
TABLE III. IR images showing the temperature distribution over time after the candles were lit when using the heat transfer system and the peak temperatures, $T_{\max }$, achieved by each shape.

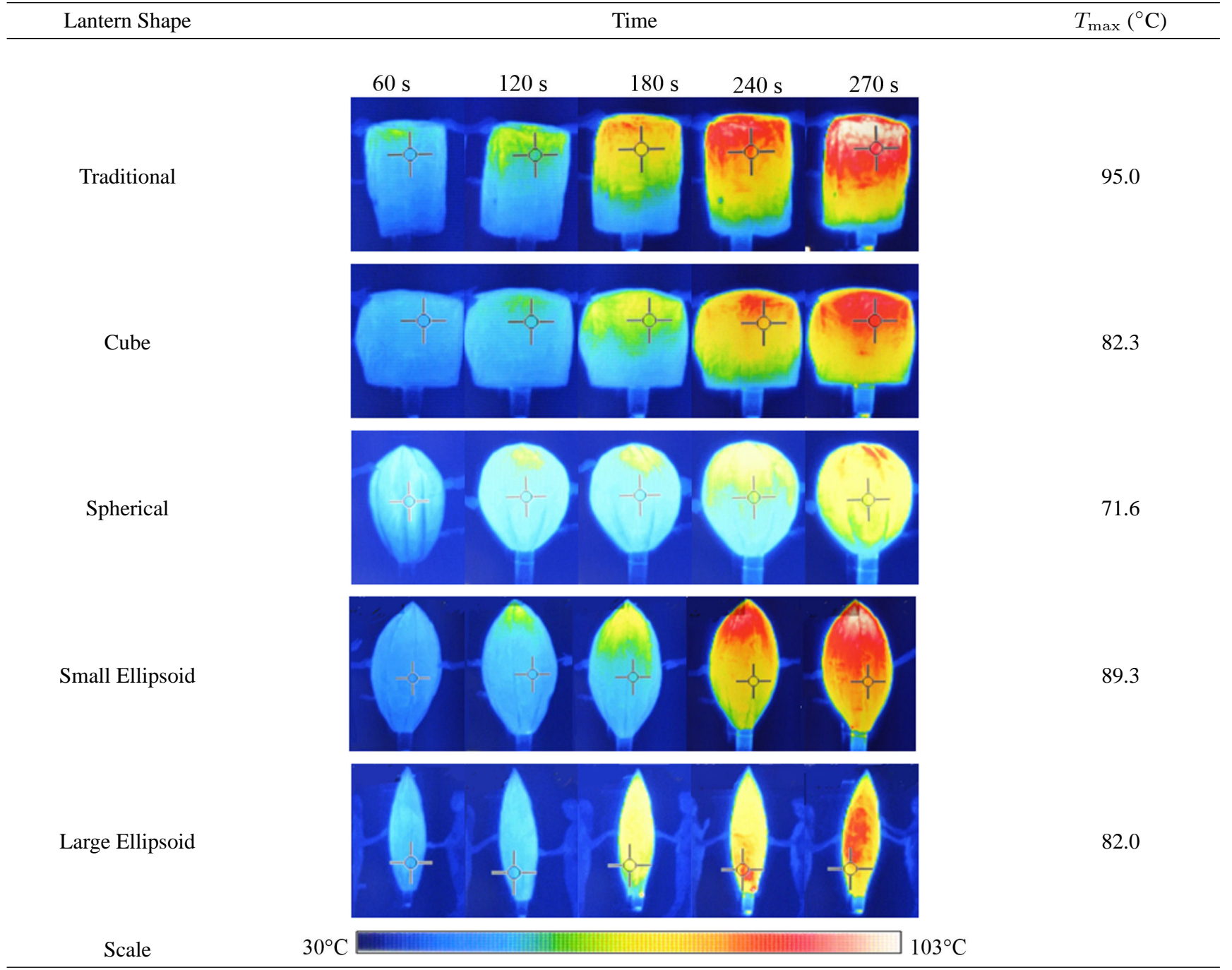

Note: Due to the large size of the large ellipsoid lantern compared to the others, the IR imaging camera had to be moved back to fit the whole lantern in the image. As a result, the lantern appears to be smaller than the others when it is in fact the largest lantern.

In general, lanterns with smaller volume required less time to transfer heat to reach $T_{\text {hot }}$. The small and large ellipsoid lanterns had almost the exact same value of $M / V$ (as shown in Table II), but the small ellipsoid lantern reached higher $T_{\max }$ than the large ellipsoid lantern. This due to the small ellipsoid lantern having less volume, therefore a smaller amount of air was required to be heated up; as such, less time was required to reach the desired $T_{\text {hot }}$ value. Another interesting result is that the traditional lantern reached the highest $T_{\max }$. This may be due to a large opening at the bottom of lantern, allowing a higher convection rate of hot air in the traditional one than other shapes [4]. However, temperatures inside the traditional lantern were unevenly distributed, meaning that it is possible that the average temperature within the lantern was in fact less than the required $T_{\text {hot }}$. As a result, the traditional lantern was unable to float to reach $2.5 \mathrm{~m}$ at the predicted $T_{\text {hot }}$ temperature. Our model should then be modified to include the lantern volume and the effect of the opening.

By allowing the lanterns to float, the time required for each type of lantern to rise $2.5 \mathrm{~m}$ was recorded and displayed alongside the amount of time taken to heat up each type of lantern to the appropriate temperature in Table IV. From the table it can be seen that the small ellipsoid lantern took the overall least amount of time to heat up and rise $2.5 \mathrm{~m}$ while the large ellipsoid lantern took the shortest amount of time to travel due to its smaller cross-sectional area and therefore lower drag coefficient. However, the overall time included the time taken to fill each lantern with enough hot air to reach $T_{\text {hot }}$, as such, the small ellipsoid lantern with the lowest $T_{\text {hot }}$ value and thorough heat distribution which allowed it to heat up in the shortest amount of time had the lowest overall time. 
TABLE IV. Time taken to heat up the lantern $\left(t_{\text {heat }}\right)$, time taken for the lantern to travel $2.5 \mathrm{~m}\left(t_{\text {float }}\right)$ and the total amount of time taken $\left(t_{\text {total }}\right)$ of each lantern.

\begin{tabular}{cccc}
\hline Lantern Shape & $t_{\text {heat }}(\min :$ second $)$ & $t_{\text {float }}$ (second) & $t_{\text {total }}($ min:second $)$ \\
\hline Traditional & $5: 23$ & 4.29 & $5: 27$ \\
Cuboid & $4: 31$ & 3.11 & $4: 32$ \\
Spherical & $7: 09$ & 5.78 & $7: 14$ \\
Small ellipsoid & $4: 00$ & 5.01 & $4: 05$ \\
Large ellipsoid & $4: 27$ & 3.04 & $4: 30$ \\
\hline
\end{tabular}

\section{Conclusions}

This paper aimed to identify the optimal conditions required for lanterns to float in the shortest time using only a tea light to heat it up. The overall time, $t_{\text {total }}$, consisted of the time taken to fill the lantern with hot air, $t_{\text {heat }}$, and the time taken for the lantern to float $2.5 \mathrm{~m}, t_{\text {float }}$. From the experiment it was found that $t_{\text {heat }}$ always had a significantly larger value than $t_{\text {float }}$, as such optimizing parameters that affected $t_{\text {heat }}$ were prioritized where paraffin candles with multiple wicks spread out over a large area and a more efficient convective heat transfer system were created, allowing the air within the lantern to reach $T_{\text {hot }}$ and therefore rise in a shorter amount of time.

The basic principles of physics in relation to buoyancy and the ideal gas law were used to derive the equation for $T_{\text {hot }}$ which is dependent on the ambient temperature, ambient pressure, and ratio of mass to volume of the object, $M / V$; as such, lanterns of different shapes were created including traditional, cuboid, spherical, small ellipsoid, and large ellipsoid lanterns. It was found that the small ellipsoid lantern required the least amount of time from the moment of the candle being lit to when it traveled $2.5 \mathrm{~m}$ vertically due to having the lowest $T_{\text {hot }}$ value as a result of also having one of the lowest $M / V$. Lanterns with even lower $M / V$ values have the potential to take an even lower overall time. However, the experiment still has standard errors in the calculation of $T_{\text {hot }}$ which could in fact result in the difference of that value between the traditional, large ellipsoid, and small ellipsoid lanterns being negligible. This could mean that there are parameters that were not considered in this experiment that could have an effect on the lantern such as the lantern volume and the size of the opening at the bottom of the lantern; this is suggested due to the similar values of $M / V$ between the traditional and the large ellipsoid yet the ellipsoid lantern can be released to float successfully at temperature closer to predicted $T_{\text {hot }}$. This suggested a modification of our theoretical model in Eq. (6) to include the effect of the opening.

Notably, this paper provided a possible solution and explanation to the ill-structured problem, so that physics teachers and educators can apply this information to teach buoyancy, lifting force and air drag. These can also be implemented to design STEM projects or activities at a high school level.

\section{Acknowledgments}

The authors would like to thank the IYPT2012 participants from the Chiang Mai University Centre. The experimental site was provided by the Department of Physics and Material Sciences, Chiang Mai University. We thank the Institution for the Promotion of Teaching Science and Technology (IPST) for their financial support. We also thank the IYPT committee for providing this creative and challenging problem.
1. D. H. Jonassen, Instructional design model for well-structured and ill-structured problem-solving learning outcomes, Educ. Technol. Res. Dev. 95 (1997) 65. https://doi.org/10. $1007 / \mathrm{BF} 02299613$

2. IYPT archive, 25th IYPT (2012) Problem 12 Lantern, Retrieved, May 10, 2016. http://archive.iypt.org/ problems/

3. M. Se-yuen and L. Chun-lun, Flying lantern sheds a brighter light on Archimedes' principle, Phys. Educ. 39 (2004) 382, https://doi.org/10.1088/0031-9120/39/5/ F03

4. Y.-T. Lin, T.-J. Yeh, and H.-S. Wu, Experimental study of convective heat transfer from the surface of a gyratory lantern, Int.
Commun. Heat Mass Transf. 27 (2000) 719, https: //doi. org/10.1016/S0735-1933(00)00152-4

5. Engineering ToolBox, Drag Coefficient, Retrieved, June 15, 2020. https://www.engineeringtoolbox.com/ drag-coefficient-d_627.html.

6. National Candle Association, Retrieved, January 17, 2012. http://www.candles.org/home_types.html

7. A. Hamins, M. Bundy, and S. E. Dillon, Characterization of Candle Flames, J. Fire Prot. Eng. 15 (2005) 265, https: //doi.org/10.1177/1042391505053163.

8. Air density calculator, Retrieved, May 10, 2020. https:// www.gribble.org/cycling/air_density.html. 